\title{
Thea: empowering the therapeutic alliance of children with ASD by multimedia interaction
}

\author{
Rita Francese $^{1}$ D $\cdot$ Michele Risi $^{1} \cdot$ Genoveffa Tortora $^{1} \cdot$ Francesco Di Salle $^{1}$
}

Received: 14 April 2020 / Revised: 21 August 2021 / Accepted: 29 August 2021

Published online: 30 October 2021

(c) The Author(s) 2021

\begin{abstract}
The Therapeutic Alliance (TA) between patient and health provider (therapist or clinician) is one of the most relevant factors for the success of a therapy. In the case of people suffering from Autism Spectrum Disorder (ASD), the alliance is extended to all the people involved in their care (i.e., teachers, therapists, clinicians, relatives). In this paper, we propose a multimedia application named Thea for empowering the TA of children with ASD by improving the communication among the TA members, sharing guidelines, multimedia contents, and strategies to comply with challenging behaviors and progress with particular attention towards end-users who are occasional smart-users. A detailed process for empowering the TA members by enhancing the informed interaction among all of them is proposed and implemented. A vocal assistant also supports patients/caregivers and therapists in documenting their activity with the person with ASD by recording videos in a free-hand modality. After a contextual analysis based on Thematic Analysis Template, Thea has been implemented using a user-centered development approach. We performed three iterations involving the end-users. A user study is performed at the third iteration. Results of the user study revealed a positive attitude towards the application. In particular, the perception of empowerment of participants increased after the tool had been used. We also highlighted the guidelines and tools that may be adopted for empowering different kinds of patients. The first results seem to suggest that the use of Thea may increase the belief of the caregivers of a person with ASD to be able to better take care of her, in a more controlled and informed way.
\end{abstract}

Keywords Autism Spectrum Disorder - Empowerment - Chatbot · Multimedia Interaction · Therapeutic Alliance

Rita Francese

francese@unisa.it

Extended author information available on the last page of the article 


\section{Introduction}

Nowadays, the term empowerment is largely adopted in various professional fields, ranging from business, health, education, and psychology. It emerged during the '70s, when Freire [15] stated that a person who has the right resources may critically perceive his personal and social situation, gain control and improve his life. This is particularly true in the health field. Many health policy programs favor patient empowerment [27] and encourage health care systems to be centered on the patient [32]. This goal is reached by adopting healthcare information systems designed to empower patients by positively influencing their behaviors that will act on their quality of life. Another factor that may improve the therapy results is the pursuit of the Therapeutic Alliance (TA). According to Horvath et al. [20], TA between patient and therapist is reached when they agree on the treatment goal and on the tasks to be performed to pursuit the goal, and reciprocal positive feelings are created.

The empowerment concept has been extended to all the family when it includes a child with a disability. This is particularly true for families with children with serious development disorders: family members have to be informed and participate in the planning and delivery of the therapy to their children [16]. The concept of TA too may be extended in this case to all the actors that take care of the child: family members, personal assistants, teachers, therapists, clinicians, and psychologists. The use of multimedia and mobile technology let us foretell future applications for moving therapy beyond the one-on-one encounter. A software system to empower the TA should first establish strict communication among all the alliance members for sharing the planning and monitoring the treatment results. This system should follow specific guidelines to be accessible to occasional smartphone users, who have different technological backgrounds and cultural levels [40].

The contribution of this paper is the following:

- propose a multimedia application, named Thea, for empowering the TA members (caregivers, patient's family members and the patient, when possible) of a person with an Autism Spectrum Disorder (ASD) by sharing guidelines, multimedia contents and strategies to comply with challenging behaviors and progress.

- design the multimedia empowerment process addressed to the TA members of children with ASD;

- ease the Thea user interaction by offering a vocal assistant to support patients/caregivers and therapists in taking videos in a free-hand modality.

- assess whether the use of Thea empowers the Therapeutic Alliance perception;

- propose guidelines and tools for the analysis of an application for empowering the TA of other kinds of patients.

The paper is structured as follows. We discuss related work and background concerning empowerment, TA and multimedia interaction in Sect. 2; Sect. 3 introduces the empowerment goals of the TA; Sect. 4 describes the proposed approach concerning the empowerment of the TA of a child with ASD. Section 5 and Sect. 6 describe the definition of the empowerment goals and the prototyping cycle of the Thea development, respectively. Section 7 discusses the lessons learned. Finally, Sect. 8 concludes the paper and proposes future work. 


\section{Background and related work}

\subsection{Patient empowerment}

In Europe, great relevance is given to patient empowerment through the European Patients Forum which promotes "the development and implementation of policies, strategies and healthcare services that empower patients to be involved in the decision-making and management of their condition...”. Empowerment is a multidimensional concept of which many definitions have been provided in the literature [5, 27]. Bravo et al. [5] designed a conceptual map of patient empowerment which has been derived through literature review and qualitative interviews with key stakeholders. The analysis identified that patient empowerment depends on two factors: behaviors and capacities which are strictly connected.

In the literature, many applications already exist supporting the patient in the autoadministration and self-monitoring of therapy [2]. In [23] an empowerment approach for people affected by diabetes is proposed. It is based on a self-management tool that enables the patient to specify his customized activities and his goals by considering medical recommendations. The usability and usefulness of that framework were also assessed.

Vitiello et al. [41] presented an empowerment methodology based on User Experience (UX) requirements. A contextual investigation is conducted to understand behaviors and capacities to be empowered and propose life quality improvements. A preliminary case study on elderly people has been discussed in [42].

\subsection{Therapeutic alliance \& multimedia interaction}

The relationship between the therapist and the patient has a serious impact on the results of the treatment [17]: it can determine treatment outcome, treatment dropout, and treatment attendance.

The alliance is composed of two factors: a "personal alliance" based on the interpersonal relationship created between patient and clinician, and a "task-related alliance" based on the sharing of goals, methods, focus, and "depth" of the treatment [21]. TA has a large impact on patient empowerment, as shown by Anderson et al. in [3]. The patient passes from being a passive recipient of care to become an active participant, establishing an equitable partnership with the clinician [18]. The patient provides to the alliance his knowledge on his status and his experience, while the clinician provides the knowledge on how to manage the patient's status. The alliance lets the patient be more responsible for his care and participate in the decisions concerning his health through the development of selfesteem, confidence, and self-efficacy [28].

In the related scientific literature, several software applications have been proposed for enhancing TA between patient and clinician. As an example, in [25] patients with eating disorders adopt an app for self-monitoring and for patient-clinician linkage, which enables clinicians to access patient data anytime. Abrahamyan et al. [1] present a platform that supports the communication among people with disability and lets the clinicians monitor patient communication, store medical records for organizing group discussions and diagnosis.

Richards et al. [36] assessed whether the use of technology would enhance patient ratings of therapeutic engagement in the case of face-to-face therapy in psychotherapy. The adopted software platform, named goACT, includes "appointment reminders, two-way 
pre-scheduled messaging (secure SMS, online, email), self-monitoring, secure journaling, online homework records, and online psychometric assessments". It emerged that the use of GoAct enhanced therapy.

Sun et al. [38] proposed a multimedia-based approach for helping caregivers and patients in the management of their self-care after a cancer intervention.

The parent-therapist alliance also highly impacts the therapy outcome for children with ASD. A family-centered intervention model is the key, with a strong emphasis on the collaboration between parents and therapists in the activities related to the planning and delivering of the therapy [10]. The de facto therapeutic role conducted by the educational institutions has been investigated in [11]. The TA between the therapist and the child is most effective when it supports the alliance of the network of relationships among therapists, parents, and teacher.

Map4speech [24] is a mobile app exploiting the Behavior Modeling Training (BMT) approach to teach the user to conduct a naturalistic intervention with children with ASD to enhance their functional communication. This application proposes predefined videos. In the app developed in our case study, all the contents are provided by the therapists and by the caregivers.

\section{Therapeutic alliance empowerment goals}

The Therapeutic Alliance is present in all treatment approaches, with some discernible differences concerning how the alliance is conceptualized. Muran and Barber [30] noted that each one of the three most diffused treatment approaches (i.e., psychodynamic, cognitivebehavioral, and humanistic therapies) considered TA to be an important treatment variable and that the difference among approaches lies within which aspects of the alliance are emphasized and utilized in the therapeutic process.

In the case of people with ASD the therapeutic alliance is extended to all the caregivers, including teachers and therapists.

Their empowerment may be reached by acting on several factors involving behaviors and capacities [5]. Figure 1 shows the capacities and behaviors which promote TA empowerment and the relationships with environmental influences. We derive them by [5] and [22]. The description of the various dimensions is provided in Table 1.

\section{The proposed approach}

Children and adolescents with neurodevelopmental disturbs may be shy, aggressive or obsessive, and have school difficulties. Many different psychopathologies are associated with these problems and all require personalized rehabilitative and educational interventions. Every child is a specific case. Only concerning the problems related to autism in the United States 1:68 child belong to the spectrum. In Italy $20 \%$ of children have learning disturbs of various origin. It becomes essential to properly take care of patients with appropriate rehabilitative interventions as soon as possible. Day-by-day rehabilitative activities must be defined. The most adopted interventions are based on the Applied Behavior Analysis $(\mathrm{ABA})^{1}$ therapy.

$\overline{{ }^{1} \text { https://www.autismspeaks.org/applied-behavior-analysis-aba-0 }}$ 


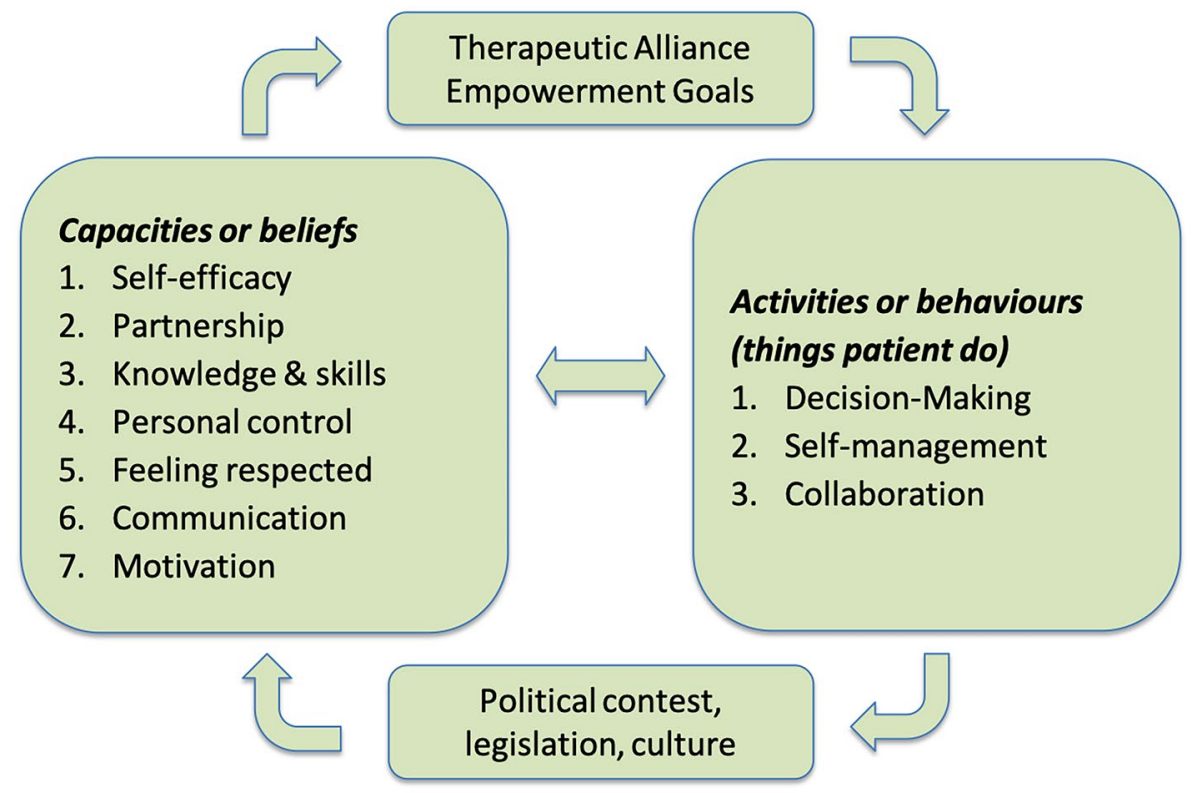

Fig. 1 Therapeutic Alliance empowerment factors

ABA therapists and experts recognize that the therapeutic relationship provides a significant contribution to patient improvement. Collaboration and teamwork are among the most relevant empowering factor [6]: therapist and client (patient or his caregivers) work together, as a team, to identify the client's problems as well as to identify solutions. The treatment plan should be explored together, by experimenting with strategies and evaluating the results, and reformulating the plan. All the people that take care of the children have to actively take part in the planning.

The goal of this paper is to propose and assess a multimedia application, named Thea, for empowering the TA members (e.g., caregivers and patient's relatives) of a child with ASD by sharing guidelines, multimedia contents and strategies to comply with challenging behaviors and progress.

User-centered design is largely adopted in e-health application development to ensure effective outcomes [37], and is counseled by the World Health Organization (WHO). We adopted the methodology shown in Fig. 2 that introduced in a traditional user-centered design methodology aspects related to the TA empowerment. In the following, we detail how the activities of the methodology have been addressed in the considered case study. In particular, the Prototyping cycle has been conducted three times.

The following two sections describe the definition of the empowerment goals and the prototyping cycle for the development of Thea.

\section{Define the empowerment goals}

The first step to perform is the understanding of the empowerment levels of the Therapeutic Alliance circle, including the provider(s) of the health service. As depicted in Fig. 2, this step is composed by several activities, described in the following. 
Table 1 Therapeutic Alliance empowerment goals

Capacities or beliefs

Self-efficacy Self-efficacy refers to individuals' assessments of their effectiveness or competency to perform a specific behavior successfully [34]. It is concerned not with the skills one has but with judgments of what one can do with whatever skills one possesses [4].

Partnership

Adopting a partnership style within the healthcare relationship lets the patients be the experts by experience, while clinicians and therapists are the experts in the knowledge of the disease.

Knowledge \& Skills

Enabling factors to be able to engage with the healthcare provider.

Personal control

Based on the person's belief that the patient has the power to produce positive results.

Feeling respected When a patient (or her caregivers) feels to be respected by the health providers communication and motivation improve. When patients (or her caregivers) are respected and they trust providers, they more likely seek care, establish a more strict relationship with the provider and follow the therapy.

Communication

The communication among the members of TA is good. They share their experience in patient support/advocacy group for sharing common goals, also through social network.

Motivation

Motivated patients increase their self-efficacy and obtain better results.

Activities or behaviors

Decision-making

Actively make informed decisions about their own health and choose realistic objectives.

Self-management

Patients have in charge their self-management behavior and adhere to a prescribed therapeutic regimen.

Collaboration

The patient works together with the TA members in pursuing mutually negotiated goals.

\subsection{User scope identification}

First, we identified the target users. They are Italian people members of a circle which cooperates in the care of a patient with ASD. Clinicians and therapists are the professionals involved, while caregivers are parents, teachers, personal assistants, and the patient when

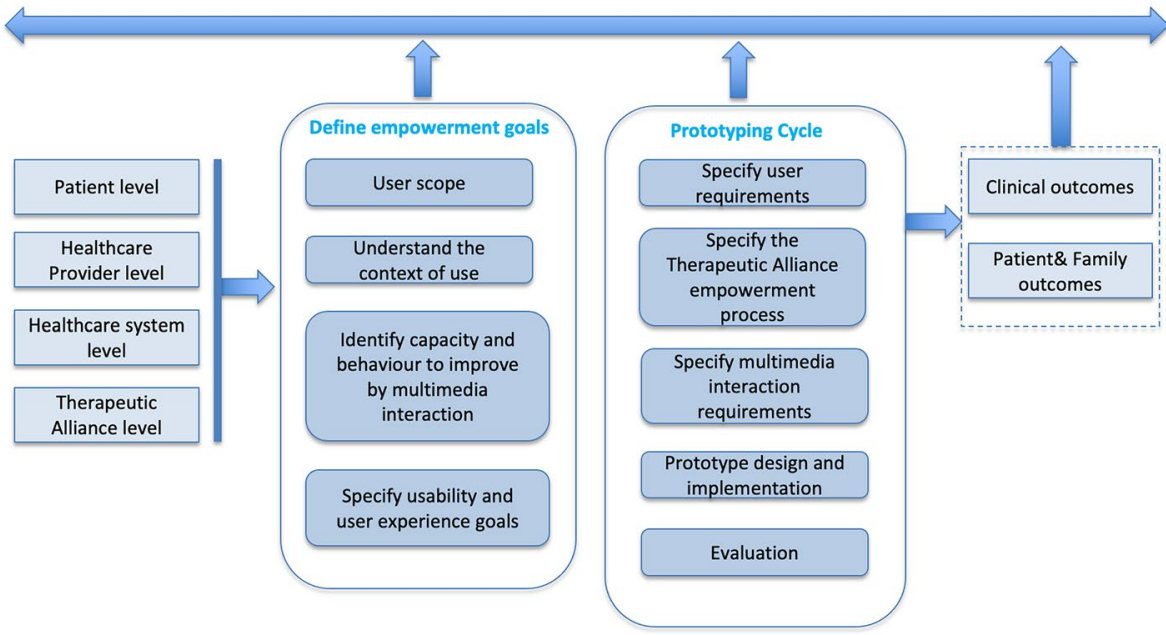

Fig. 2 The adopted user-centered methodology 
autonomous enough. The target users are of different technological backgrounds and social environments.

\subsection{Understand the context of use}

The understanding of what the domain of the community to be empowered is may be performed by querying the interested people on which the process of managing their care is and how it is conducted. This phase is composed of two steps:

1. Interviews for caregivers and professionals, where we selected some users representative of each TA user category, proposed a demographic questionnaire concerning gender, cultural level, employment, and technological level and conduct a semi-structured interview useful to get feedback and write a survey;

2. survey, dispensed to several users of each category to understand how the aspects that the interviewed users highlighted are actually managed, what is wrong and what is positive, and which their empowerment level is.

\subsubsection{Interviews for caregivers and professionals}

Method. We decided to divide the members of the TA of a child with ASD into two groups of users: caregivers (family members, teachers, personal assistants) and professionals (therapists, clinicians, psychologists) and designed an interview for each group. We adopted Thematic Analysis Template (TAT) for structuring the interviews, a qualitative method to identify patterns, themes, and interpretations in data [29]. TAT uses template documents, which list a set of initial themes that have to be discussed.

Study material. We designed two templates: the template in Table 2 for the caregivers and the one in Table 3 for professionals. The templates were manually filled in by all the researchers, who all agreed on the selected themes and also registered the discussion.

Interview to caregivers. We interviewed two mothers (M1 and M2), two teachers (T1 and T2), and a personal assistant (PA). The interview was conducted by following the template in Table 2. The following findings were collected. The ids of the questions we proposed in the survey described in Sect. 5.2.2 are associated in bold to each relevant sentence.

Shared decision-making and integration of patients' views.

a. The general procedure of take care and subministrate the therapy is the following: the ABA expert performs a first meeting with the parents and evaluates the child through standard tools. Successively, the expert defines an operating relationship which is shared and discussed with the therapists, the school, and the family. Therapists then work with the patient for the weekly established number of hours. The treatment is monthly monitored, through video and data.

b. When the procedure is correctly actuated, parents share the goals with the ABA expert as follows: after having evaluated the child the expert asks the parents what are the things the daughter prefers to do. Teachers generally are involved in the meeting via Skype. PA is informed by the child's mother on the activity to do and informally by the therapist when he meets him.

c. The initial goals should be established by involving all the caregivers. The goals are divided into the various cognitive areas, communication, social skills, and academics, 


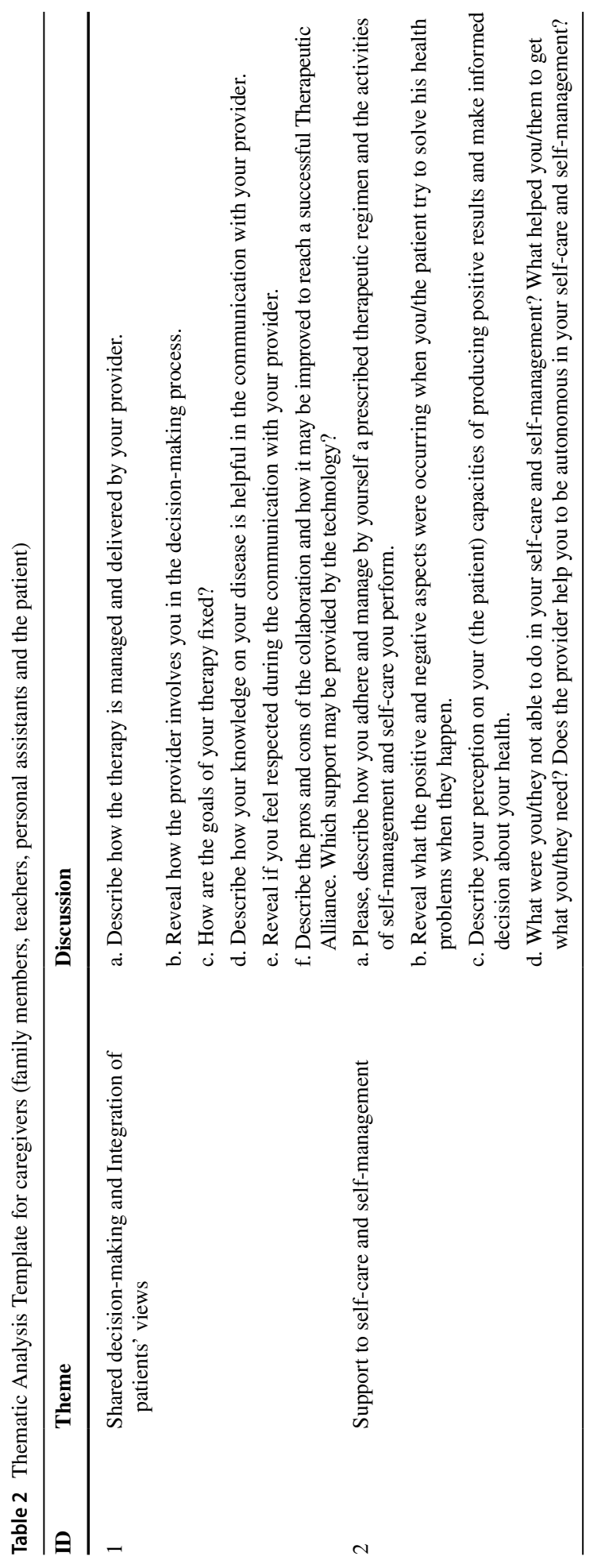




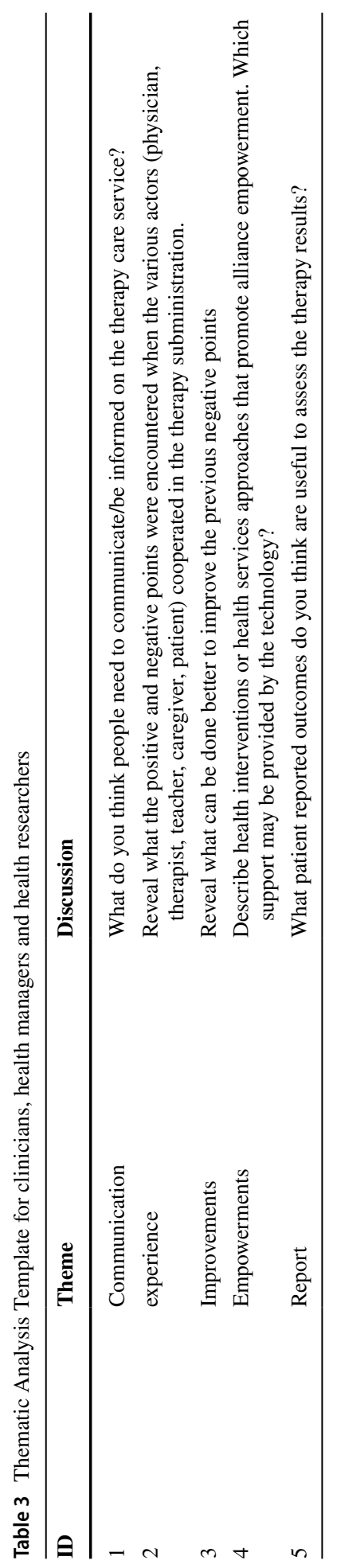


autonomy development, such as fine motor dexterity, domestic capabilities, grooming, hygiene, and competence at work.

d. All the interviewed agreed that the background is fundamental for the self-management of their children and also for discussing with their providers.

e. The daily communication among the circle members generally occurs via Whatsapp (Q6). Caregivers have to take a diary where they annotate the progress, the reached level of autonomy, and the challenging behaviors $(\mathbf{Q 1 0})$. Rarely therapists produce videos of the therapy. M1 would like something to explain to the other family members how to interact with her daughter. T1 would like to be updated more frequently on the progress and the problems of the child (Q5). M2 says that when a therapist or a teacher is substituted, the new entry is not informed on the child's problems and does not know how to manage her daughter, therefore producing negative effects.

\section{Support to self-care and self-management.}

a. Once the patient level has been assessed, the goals are identified and for each goal, a list of tasks is defined. Each task is then analyzed and subdivided into its components. After having learned a competence in the controlled setting of the therapist, ABA is extended to the real settings, such as home, school, and external environments. So the caregiver follows the guideline by successively executing each step of each task.

b. One of the mothers follows the videos she has taken while the therapist manages her daughter and spreads them to the teacher and her Personal Assistant (Q9). To try to understand what causes a challenging behavior (e.g., self-injury) and how to prevent it, parents have to collect information related to the problem behavior and understand antecedents (what happens before a behavior occurs) and consequences (what happens after the behavior). Parents have to complete the ABC chart every time a problem behavior occurs (Q11). See [14], where an example of the ABC Chart is shown. The challenging behavior occurring has to be communicated to the other people of the circle (Q5). To this aim they generally use Whatsapp.

c. People who are better formed are more aware and able to make an informed decision and to have a positive influence on the child's results since they always actively participate in the therapy planning. Generally, the participants perceived that their opinion is taken into account.

d. Participants would like to have more precise guidelines and more activities to perform (Q9); more contact with the provider (Q12). The use of Whatsapp for communicating merges different kind of information, including greetings, and make difficult to search the data.

Interview to medical staff. The interview has been conducted by following the template in Table 3. We involved a neurologist, a psychologist, and a therapist of private centers. Results are summarized in Table 4.

\subsubsection{Survey}

Method. The interviews conducted by using the TAT analysis are transcribed and the most relevant aspects are identified. Then, a question is associated with each relevant theme, identified by the question id in bold in Sect. 5.2.1. 


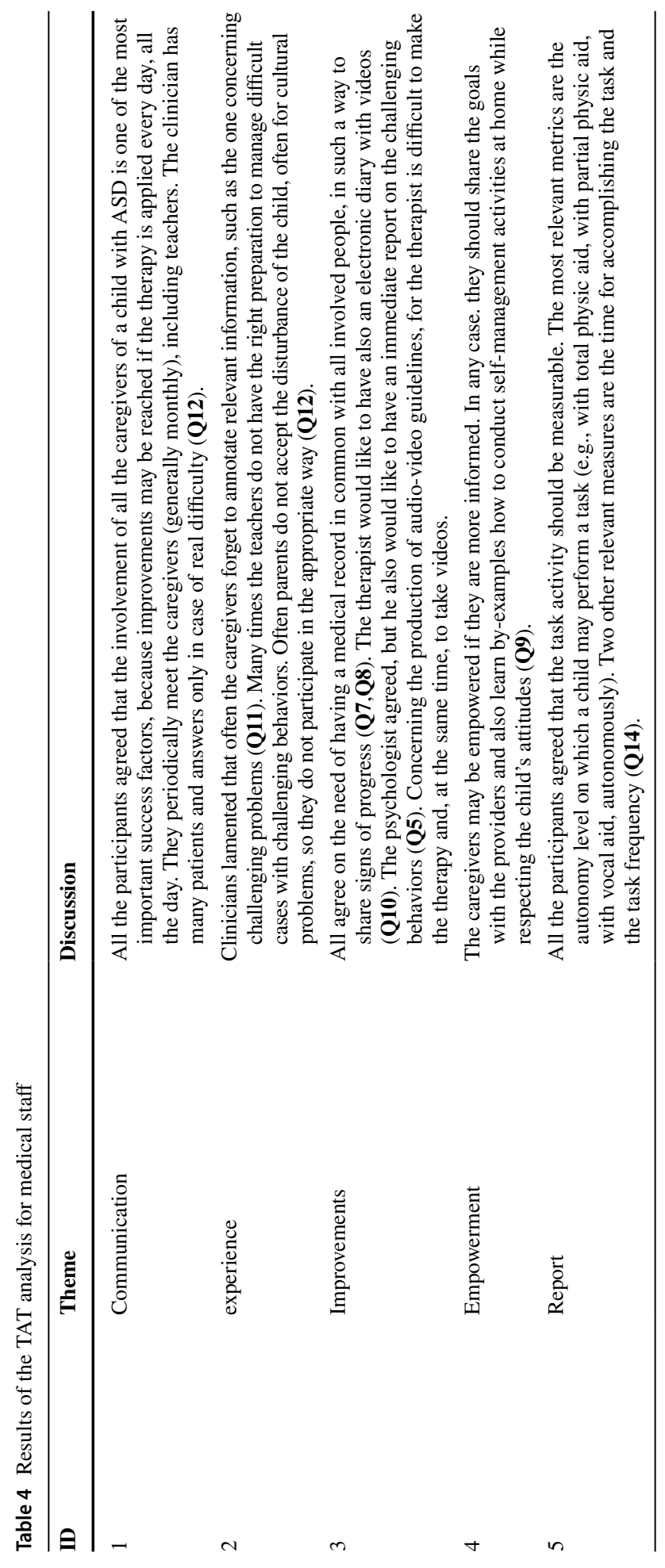


Table 5 Questionnaire for the Therapeutic Alliance actors of a children with ASD

\section{ID Question}

Q1 I use the smartphone for communicating with the other people involved in the care of the special needs person.

Q2 I use emails for communicating with the other people involved in the care of the special needs person.

Q3 I use apps (e.g., Whatsapp, Messenger, Instagram, Facebook) for communicating with the other people involved in the care of the special needs person.

Q4 I use Face-to-face for communicating with the other people involved in the care of the special needs person.

Q5 It is useful to promptly communicate with the other people involved in the care of the special needs person.

Q6 It is useful to use a single means of communication.

Q7 It is useful to have the patient's information needed for a diagnosis all in a single place.

Q8 It is useful to have the material to evaluate the patient progress in a single place.

Q9 It is useful to have at disposal examples of the activity conducted during the therapy for all the caregivers.

Q10 It is useful to have an electronic diary where to annotate the challenging behaviors.

Q11 It is useful to collect data to compile the ABC table "antecedent- challenging behavior - consequent".

Q12 I'm satisfied of the communication between the people that take care of the special needs person.

Q13 If no, describe these problems (open question).

Q14 (only for clinicians and therapists) Which task progress metrics do you consider relevant? (suggested: Autonomy Level, Frequency, Time to accomplish a task, more than one answer is allowed and additional may be added).

Study material. We prepared the questionnaire shown in Table 5. The answers were scored with a Likert scale from 1 (not at all) to 5 (very much). We also collected demographic information of participants. Questions Q1-Q4 are devoted to the understanding of which communication means the participants usually adopt for communicating with the alliance members. The other questions are derived from the TAT analysis to further verify their real needs.

Study context. Participants were 101 end-users belonging to associations of family members with an ASD child, $65.5 \%$ woman, the other men. Their roles in the patient alliance were: $31 \%$ family members, $27.6 \%$ psychologists, $13,8 \%$ therapists, $10.3 \%$ teachers of children with special needs, $6.9 \%$ personal assistants, and $3.4 \%$ clinicians. $65.5 \%$ were graduated. All the users had a smartphone and used Internet. 31\% made large use of social networks, $17 \%$ made use of e-Commerce for buying products, and $27 \%$ read online news. Only $13 \%$ played online games. $44.8 \%$ were accustomed to installing and using new apps on their own smartphone. So we can conclude that most of them moderately used a smartphone.

Results. The histograms in Fig. 3 summarize the answers provided to the questionnaire in Table 3. Concerning the means participants use for communicating with the other members of the circle, 46\% uses the phone (Q1), email only by $9 \%$ (Q2), 51\% uses Whatsapp (Q3) and 68\% communicate face-to-face (Q4). 72\% consider useful to have the possibility of immediate communication with the circle members (Q5), while $67 \%$ judges useful to employ only one of the communication means (Q6). Also to collect all the material in a single place for diagnosis purpose (and for evaluate the patient progress) is considered useful by $84 \%$ of participants (Q7) (93\% Q8). Most 


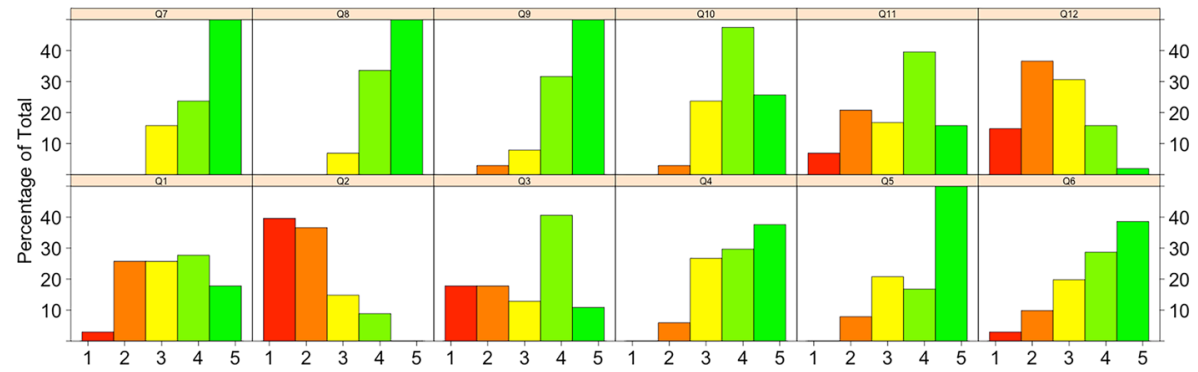

Fig. 3 The survey results

participants (89\%) were favorable to the idea of having examples of the therapy subministration available for all the circle (Q9). 73\% of participants agreed on the need of having an electronic diary at disposal where annotating the challenging behaviors (Q10), while the $55 \%$ of them were interested in filling the ABA information (Q11). Only $18 \%$ was satisfied by the communication among the people involved in the patient care $(\mathbf{Q 1 2})$. The open question $(\mathbf{Q 1 3})$ collects their concerns: some clinicians and therapists scarcely communicate the therapy objectives and the way to reach them and the caregivers have a sense of loneliness. Some of them do not take part in the therapy planning. They signal the lack of group sessions with all the involved parts and the lack of information of the clinicians on the patient status. On the other side, therapists and clinicians lamented having a very high workload with a lot of patients and that often patients do not follow indications and do not have the skills for providing a useful contribution. Concerning the task performance metrics $(\mathbf{Q 1 4})$, all the clinicians agreed that the three suggested metrics are essential.

\subsection{Identify capacity and behavior to empower with multimedia interactions}

Table 6 shows the relevant tasks we identified from TAT interviews and the questionnaires. The table also shows for each task the behaviors that are modified by the process and the capability which should be improved. We also identified which activities need the support of multimedia features.

The analysis of the contextual inquiry revealed that $\mathrm{T} 1$ is a task that has to be mainly conducted in F2F modality, or in video-conference, while the other tasks may be supported by multimedia technologies. The therapy of each child is customized in terms of tasks and measured in terms of autonomy level, frequency, and accomplishing time of each task. So pre-defined material may be not appropriate for each circumstance. The idea of providing video/audio guidelines created ad-hoc by the therapist appears to be the most appropriate. Similar functionalities may be adopted at home or at school.

\subsection{Specify u sability and user experience goals}

Before starting development, Usability and User Experience goals have to be defined by taking into account that people involved in the TA may be occasional smartphone users and with different cultural backgrounds. Thus, this kind of application has to be particularly 


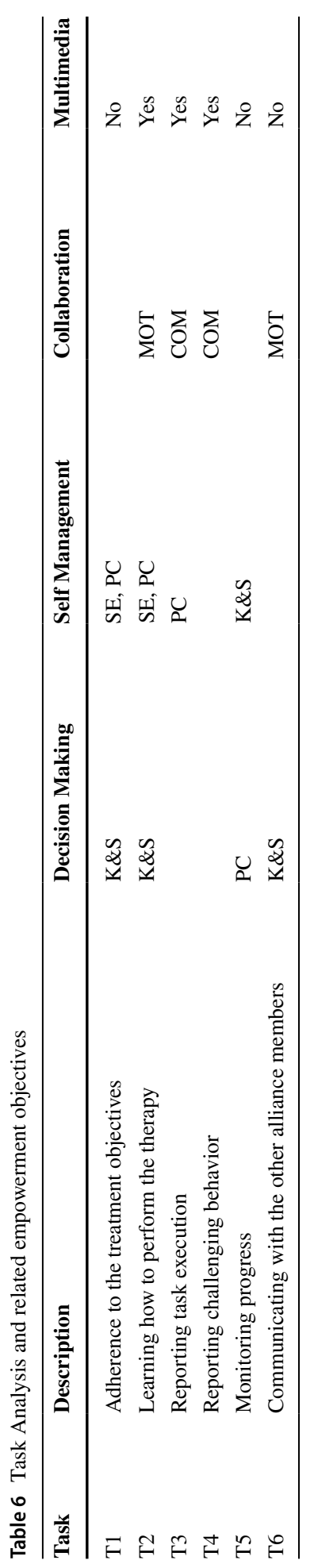


easy to use to avoid the risk of abandonment. Also, clinicians and therapists have to find it appropriate to use during clinical practice. So, also for them, there is the need to immediately find the required functionality to save time. We identified the following usability goals for all the actors: (1) Effectiveness, the application has to provide a guide for the Thea members and help them in the therapy activities; (2) Efficiency, the application has to help the caregivers in performing the patient tasks efficiently; (3) Satisfaction, the application should be judged easy to use and helpful by every Thea user of whatever background. We also are interested in evaluating the global User Experience as relevant evaluation criteria. These goals have to be pursued especially for occasional users in the use of smartphone since the technological background of many TA members is low.

We decided to adopt for Thea a minimalist interface style for easing the interaction. Indeed, the app should be designed for limited and split attention [39], because when the circle members are involved in activities with the child with ASD they should be able to multitask. Cognitive aids should also be particularly considered, e.g. to remind a user of what she has to do because the attention of the users may be limited or they may be stressed. Concerning the interface colors, we decided to follow the guidelines traced in [8] that recommend exposing end-users to blue color which positively impacts on psychological effects and social interaction. We understood that it is very relevant to minimize the number of interaction points, especially when recording challenging problems (caregivers) or during the therapy recording (therapists, caregivers) where the end-user attention is addressed to the child. User registration should also be minimal, we do not expect family members having a lot of problems to spend a lot of time in data entering. The use of a usercentered design approach should enable us to get better human-computer interaction.

\section{Prototyping cycle}

In this section, we describe three prototyping iterations. The first is centered on the design, the others mainly on development activities.

\subsection{First Iteration}

Specify user requirements. We conducted a focus group with the same users involved in the interview, who were informed that all the discussions related to the study were confidential. The objective of this session was to identify the list of requirements to empower all the TA members in such a way to adopt the same procedure for managing the patient and conducting her rehabilitation activities with a uniform approach. We started from the

Table 7 First iteration requirements

\begin{tabular}{ll}
\hline Actor & Description \\
\hline Clinician & $\begin{array}{l}\text { manage the diagnosis data, the objectives and describe the challenging problem of a } \\
\text { patient. } \\
\text { manage the tasks to be performed;provide guidelines on how to perform each task. }\end{array}$ \\
$\begin{array}{l}\text { Therapist } \\
\text { Therapist, caregiver }\end{array}$ & $\begin{array}{l}\text { collect reports on the performed activities;provide reports on challenging behaviors. } \\
\text { examine the patient record;monitor signs of progress; communicate on a Therapeutic } \\
\text { Alliance common chat; receive notifications when events happens; participate in a } \\
\text { community of users for sharing experiences. }\end{array}$ \\
\hline
\end{tabular}


Table 8 Visibility types

\begin{tabular}{ll}
\hline Actor & Description \\
\hline All & All the people accessing Thea may see the user data. \\
Health providers & $\begin{array}{c}\text { The data will be available to all the providers registered } \\
\text { in the system for further analysis. }\end{array}$ \\
Circle & Data are visible only to the members of the Circle. \\
\hline
\end{tabular}

empowerment goals summarized in Table 6. This session produced as a result the list of requirements reported in Table 7 .

One of the objects of discussion was related to the privacy of the contents: participants all agreed that they would like their data to remain visible only to the circle. When we insisted by explaining that information may be useful for statistical purposes or for helping others in case of success stories some of them said to be available to share their data only with medical staff. So we decided that a user may decide to choose among three different types of visibility, as reported in Table 8.

Specify the Therapeutic Alliance Empowerment Process. By considering the empowerment goals, the inefficiencies, and the identified requirements, during the focus group we also designed the patient management process related to TA of children with ASD, in such a way to empower the participation of all the members of the alliance.

Method. We adopted a scenario-based description supported by a model of the process represented by an activity diagram with object-flows and swimlanes, where the $<<$ Multimedia $>>$ stereotype is adopted for denoting an activity which requires a more specific multimedia interaction design.

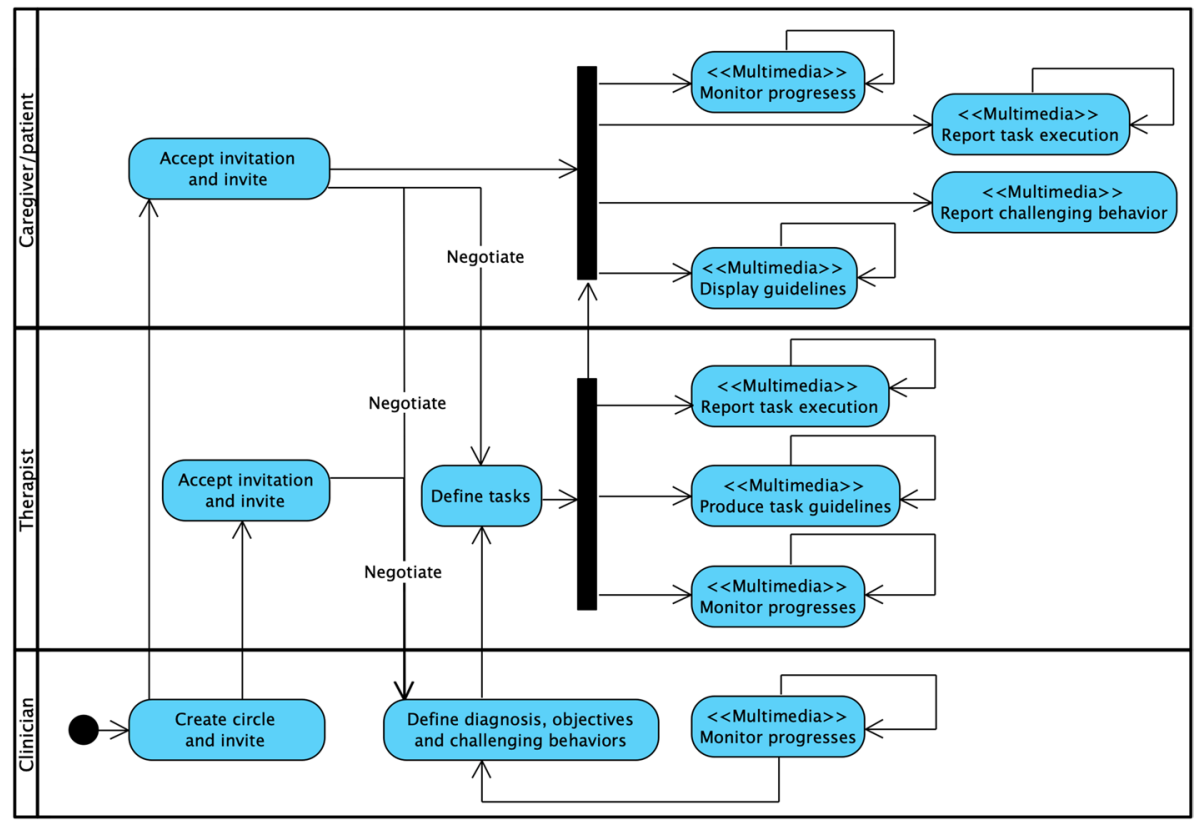

Fig. 4 Therapeutic Alliance empowerment-driven process 
Process design. We provided a scenario-based description of the process (reported in Fig. 5) and the activity diagram in Fig. 4 representing the articulation of the process in activities. We adopted a repository-based approach: all the information of a patient (the patient record) flows in a unique repository which is shared among all the TA members.

Specify multimedia interaction requirements. Method. We compiled a form for each multimedia task identified in Fig. 4. The task is modeled by an activity diagram with object-flows and swimlanes. Table 9 provides an example of task specification and the associated activity diagram is shown in Fig. 6.

Interaction modality. We decided together with the end-users to use both the traditional touch-based interaction and a female chatbot named Thea for providing support to the member of the circle when managing multimedia content during therapy. A female-gender chatbot has been chosen because women are considered friendlier [19].

Prototyping. During the focus group, we designed an initial prototype to better support the requirement collection. It has been shown to the participants when all the suggestions were collected, for not to influence them. Some of the final screens resulting from the session are shown in Fig. 7. Screens are in Italian because they are devoted to Italian people. The scale for measuring progress was also better defined (autonomy grade, time to accomplish the task, and frequency). Participants did not appreciate the dark background and asked to see a white version.

Let Hector be a child with ASD. His TA is composed of a clinician, a therapist, the school medical team (his health providers), and his caregivers: a personal assistant, the parents, and a teacher.

The clinician creates the circle with the collaboration of the Hector's mother, in the bottom swimlane in Fig. 4, and invites the other members. After in loco screening activities, the clinician specifies the diagnosis, autism with disruptive behavior, and the high-level objectives, such as improve the child's self-care and social life. These objectives are negotiated in a F2F meeting with the other members of the TA, someone may participate in remote mode. Once the goals have been individuated, the therapist (intermediate swimlane in Fig. 4) better customizes the therapy by considering his observations and the caregiver's suggestions which take into account the child's attitudes. He defines a detailed list of specific tasks that can be pursued, such as learn to brush his teeth without any help. Once the tasks are clear to the caregivers, he also produces the guidelines to suggest how this objective may be reached. He can do this by registering a vocal message, taking pictures, or also by taking a video of Hector while he is performing the task. An initial list of the current challenging behaviors of Hector is also provided after interviewing Hector and his family. All the members of the alliance may execute now the tasks and update the progress Hector performs (Report Task Execution activity). Multimedia contents may also be added to the Hector's data to share the work done with the other members of the circle. When the caregiver is not sure how the task should be performed, she can consult the guidelines and the work performed by the other circle members (Display Guidelines). The occurrence of problem behavior is also communicated to the members of the circle that subscribed to this kind of event and also store the date and the duration data of the episode. The caregivers may add other information related to the occurred episode (e.g., make a video, add pictures, fill in the antecedent-consequence table).

The material posted by the alliance is periodically checked by the clinician (Monitor progress), who consequently may modify the diagnosis and the high-level objectives.

Fig. 5 Scenario of the Therapeutic Alliance empowerment-driven process 


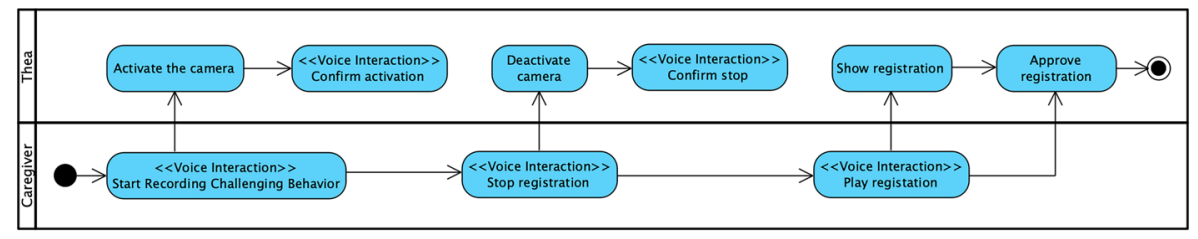

Fig. 6 Model of the Challenging Behavior Reporting process

\subsection{Second iteration}

The second prototype has been developed taking into consideration the suggestions collected during the previous interaction. Details are summarized in Table 10.

\subsection{Third iteration}

The third iteration is mainly concentrated on the improvement of the user interaction, as reported in Table 11. The prototype evaluation is deeply described in the next section.

\subsection{Third prototype user study}

We conducted a lab-based user study for the third Thea prototype in which we investigated the following main Research Question (RQ1):

RQ1: does the use of Thea empower the Therapeutic Alliance perception of caregivers?

To assess this question we investigated if there exists an effect (either positive or negative) of the use of Thea on the perceived Capacity-Belief of the participants. We also aim at assessing the Usability and the User Experience of participants globally and concerning their capability of using smartphones. So we investigated also the following research questions:

RQ2: does experience in using a smartphone impact the usability of Thea?

$R Q 3:$ does experience in using a smartphone impact the User Experience of Thea?

Table 9 An example of multimedia task description

\begin{tabular}{|c|c|}
\hline Item & Description \\
\hline Task & Report challenging behavior activity. \\
\hline Actor. & Patient/caregiver/therapist. \\
\hline Goal. & Report challenging behavior. \\
\hline Control & $\begin{array}{l}\text { The user controls the challenging behavior recording by interacting with a vocal chatbot. Also } \\
\text { the mobile device GUI supports this operation. }\end{array}$ \\
\hline Context & Patient's home or in an external environment. \\
\hline Modeling & $\begin{array}{l}\text { The activity diagram with object flow describing the multimedia interaction of the Report Chal- } \\
\text { lenging behavior is reported in Fig. } 6 \text {. The actors are the caregiver and the chatbot Thea which } \\
\text { may display multimedia content on the user's smartphone or a TV. }\end{array}$ \\
\hline Tool & $\begin{array}{l}\text { A Google Home communication point may be positioned in each room together with a webcam. } \\
\text { The interaction may also occur through a Google assistant on a mobile device. }\end{array}$ \\
\hline Feedback & $\begin{array}{l}\text { Caregivers appreciate this solution a lot for collecting data on challenging behaviors which } \\
\text { allows them to maintain their hands free for intervening. }\end{array}$ \\
\hline
\end{tabular}


Fig. 7 First prototype screens

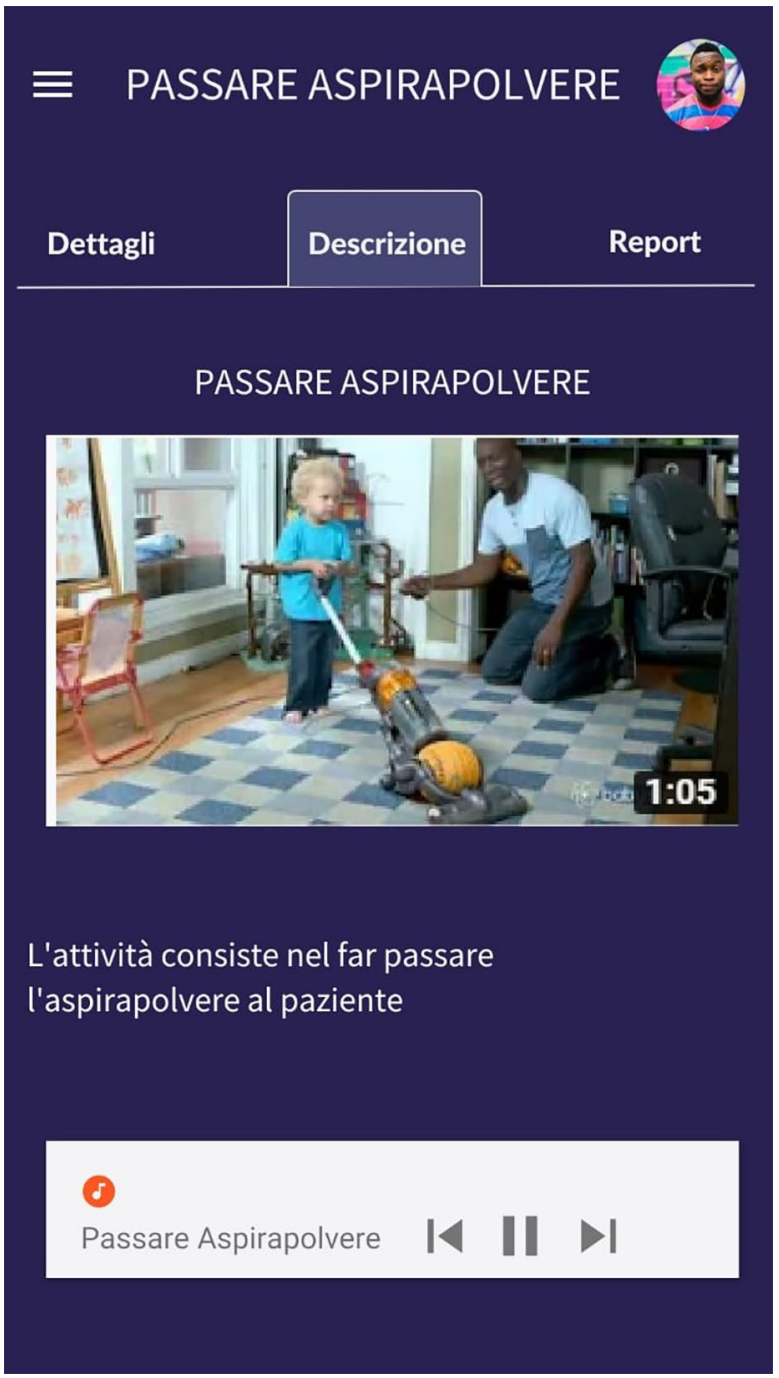

\subsubsection{Experimental units}

Participants were 31 caregivers who voluntarily took part to the study. They were all caregivers of a child with ASD. They were recruited through associations of families with an autistic child. We collected demographic data and information on participant's technological skills through a pre-questionnaire. The average age of the caregivers was 45 (11.3 standard deviation). The pre-questionnaire revealed that 18 were skilled smart users (they frequently used the smartphone for sending emails, taking videos, surfing on Internet, e-Commerce, watching TV and listening to the radio, making video calls, installing new apps), the others were occasional smartphone users (they mainly used the smartphone with a chatting app and for making telephone calls, rarely used the device for surfing). 13 of them had some college education, one had a Ph.D., the others had a Bachelor's degree. 
Table 10 Second iteration report

\begin{tabular}{|c|c|}
\hline Activity & Description \\
\hline Prototyping & $\begin{array}{l}\text { A running prototype has been developed using } \\
\text { the Angular-Ionic-Cordova technology for the } \\
\text { development of the mobile application. Ruby on } \\
\text { Rails and PostgreSQL are the adopted server-side } \\
\text { and DBMS technologies. The choice of using } \\
\text { multi-platform technology was due to the need of } \\
\text { having the application available in both Android } \\
\text { and iOS in a short time. }\end{array}$ \\
\hline Evaluation & $\begin{array}{l}\text { A heuristic evaluation was conducted with a group } \\
\text { of } 4 \text { HCI experts of the University of Salerno, } \\
\text { two of them were parents of a child with ASD, } \\
\text { and a therapist. The therapist asked to be able to } \\
\text { document challenging problems also by audio. } \\
\text { Concerning usability, some buttons were not } \\
\text { homogeneous. Some participants manifested } \\
\text { difficulty in understanding without help in the } \\
\text { use of the application. The colors depicting the } \\
\text { levels of a challenging problem were too marked. } \\
\text { Some functionalities were difficult to find and this } \\
\text { increased the cognitive load. }\end{array}$ \\
\hline
\end{tabular}

\subsubsection{Experimental material and tasks}

As an experimental object, we used the third version of the Thea prototype. It has been pre-charged by the data of a circle composed of four caregivers, a clinician, and a therapist. Tasks related to personal care have been added with the associated guidelines.

To collect the user capacity-belief perceptions we used the Questionnaire in Table 12, for the assessment of the Empowerment perception, derived by [22]. Answers are rated on a 9 point Likert Scale from 1 to 9 (strongly disagree to strongly agree). The reliability of the modified questionnaire is assessed in Appendix A.

The lab was equipped with a Philips Smart TV 7304 and a Reolink Argus 2 webcam. Thea was running on a Samsung S10e Android mobile device.

As experimental tasks, the caregivers had to perform the tasks in Table 6. They received a sheet on which the description of the tasks were reported. An example of task description is reported in Table 13.

Table 11 Third iteration report

\begin{tabular}{|c|c|}
\hline Activity & Description \\
\hline Prototyping & $\begin{array}{l}\text { To assist caregivers in the use of the app, we introduced the chatbot not only for the } \\
\text { management of multimedia content during therapy but also as an app assistant, i.e., } \\
\text { to explain the app functionalities and help the user in finding the needed features. The } \\
\text { chatbot clearly says which tasks it can perform. These tasks should be simple and not } \\
\text { ambiguous. We enable people to both interact by the GUI and the vocal input. The } \\
\text { chatbot starts by pressing a button always available on each screen of the application or } \\
\text { by saying "Hey, Thea". When the user dictates a command, the chatbot chat is opened } \\
\text { and the dialog is traced (for example see Fig. 8c). Automatic message reply is done by } \\
\text { a rule-based chatbot based on Dialogflow. }\end{array}$ \\
\hline Evaluation & A lab-based user study has been conducted (see Sect. 6.4). \\
\hline
\end{tabular}

https://dialogflow.com 

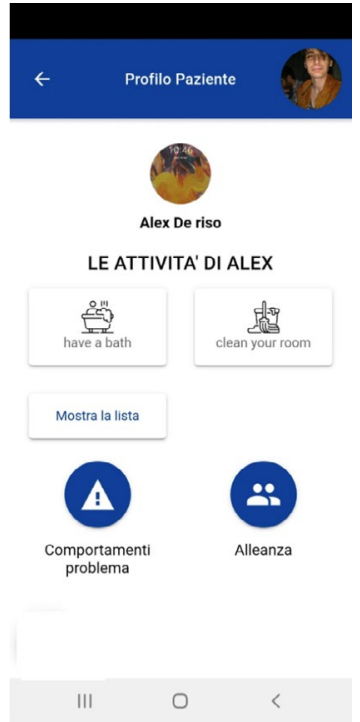

(a)
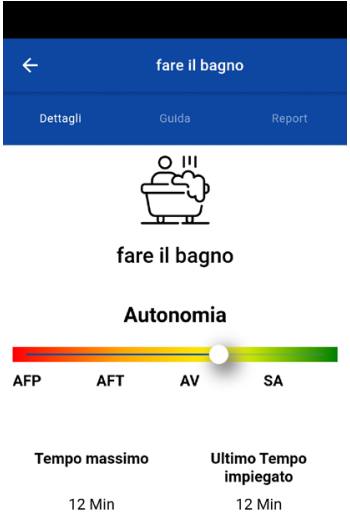

STORICO ANDAMENTO

(b)

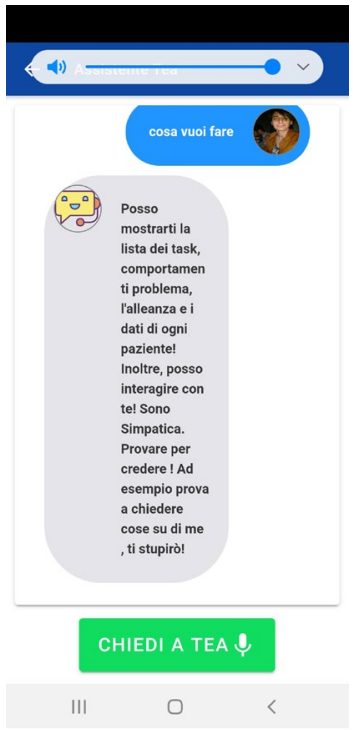

(c)

Fig. 8 Second prototype iteration (a) and (b) and the chatbot added in the third iteration (c)

After each task, to measure task-performance satisfaction, the sheet proposed the following question, which is scored on a 7-step Likert scale ranging from "very difficult" to "very easy".

\section{PT. How difficult or easy did you find this task?}

For collecting the User Experience perception we selected the UEQ questionnaire [35], composed of 26 items grouped into six scales: Attractiveness (AT), Perspicuity (PER), Efficiency (EFF), Dependability (DEP), Stimulation (ST), and Novelty (NOV). Attractiveness concerns the aesthetics of the application, Perspicuity represents the easiness of understanding the application, while dependability concerns how the app seems trustworthy. Stimulation represents the joy of using the tool and novelty describes the perception of how innovative is the product. Each item has assigned two terms, such as fast/slow. Items may be scored by using a 7 point Likert scale, ranging from -3 (fully agree with the negative term) to +3 (fully agree with the positive term). The original User Experience

Table 12 Capacity-Beliefs Empowerment Questionnaire

\begin{tabular}{ll}
\hline ID & Question \\
\hline S1 & I am sure to be able to solve my therapeutic tasks (Self-Efficacy). \\
S2 & I have an active partnership with my Therapeutic Alliance group (Partnership). \\
S3 & I have enough information for managing my therapy (Knowledge \& Skills). \\
S4 & I am sure to be able to solve my therapeutic tasks (Personal Control). \\
S5 & I feel to be accepted and well supported (Feeling Respected). \\
S6 & I am satisfied of the communication with my Therapeutic Alliance group \\
S7 & (Communication). \\
\hline
\end{tabular}


Table 13 Example of task set for T2 - Reporting Task Execution

\begin{tabular}{ll}
\hline Task & Description \\
\hline T4 & $\begin{array}{l}\text { Imagine you are in your dining room with your child. Your child is manifesting a challenging } \\
\text { behavior. Ask Thea to register the video through the webcam, consult the registration on TV } \\
\text { and approve it for saving. }\end{array}$ \\
\hline
\end{tabular}

Questionnaire $^{2}$ was in German, but there are available translations in 20 other languages, also in Italian. We adopted the Italian version. We also add an open question for free-handing comments.

\subsubsection{Hypotheses and variables}

Concerning user capacity-belief perceptions we considered an independent variable named Order, a categorical variable with two values: PRE and POST collected by subministrating the same questionnaire in Table 12 before and after the experiment.

A dependent variable is considered for each capacity-belief: Self-Efficacy (SE), Partnership (PAR), Knowledge \& Skills (KS), Personal Control (PC), Feeling Respected (FR), Communication (COM), Motivation (MOT).

- $\mathrm{HO}_{X}$ : There is no statistically significant difference between the PRE experiment and POST experiment to $\mathrm{X}$.

where $\mathrm{X}$ is one of the considered dependent variables. Because we could not postulate an effect of the use of Thea in a specific direction on the perceived empowerment of TA, either positive or negative, our alternative hypotheses are two-tailed.

Concerning the measures of Usability, we considered as an independent variable the use of smartphone experience, a categorical variable with two values Not Smart User (NSU), Smart User (SU). We follow the approach proposed by Ferreira et al. [12] and considered as dependent variables:

- Effectiveness, computed as the percentage task completion by a participant.

- Efficiency, measured as: (i) Speed: time (measured in seconds) a participant takes to accomplish the task. The time taken to display-record activity is not considered; (ii) Interactiveness: number of clicks (or question to the chatbot) made by a participant to complete the task.

- Satisfaction, represented by the average of the answers of each participant to the Post-Task question PT.

To measure user experience, we adopted a dependent variable for each UEQ category. We compute the average of the questions belonging to a given scale for each participant [35].

${ }^{2}$ https://www.ueq-online.org 


\subsubsection{Procedure}

The evaluation procedure has been conducted in the one-to-one modality in a lab setting under controlled conditions. A supervisor recorded the sessions without intervening. Participants were first informed about the aim of the study. Before starting the experiments, participants filled in the questionnaire in Table 12, to collect their perceptions on their capacities and beliefs. Participants individually received a short tutorial on the Thea functionalities. Then we let them explore the prototype for four minutes. Participants were then given tasks, detailing the ones in Table 6 to complete. The successful completion of each task was assessed by the supervisor, who observed how the participants completed the assigned tasks, took the time, and the number of interactions. After each task, the post-task question PT was answered. Upon completion of the tasks, participants were asked to fill in again the questionnaire in Table 6, the UEQ questionnaire and an open comment question.

\subsubsection{Analysis procedure}

The considered questionnaire has been extracted from a validated one. We analyzed whether the questionnaire validity has been preserved as described in the Appendix. We adopted the paired two-sided Mann-Whitney $U$ test to find out whether there is a significant difference between capacities and beliefs perceptions with the questionnaire in Table 12, before and after the experiment. In case of statistically significant difference, we apply the Cliffs distance [26] for determining the dimension of the difference. We also used boxplots to illustrate the score distributions for each response variable and report the statistical data. A similar procedure is adopted for the Usability and User Experience variables for which we compare the statistical data of SU and NSU to verify if there is a difference in their perceptions.

Table 14 Statistics of the Capacity-Belief questionnaire

\begin{tabular}{lllllll}
\hline Technology & Variable & Min & Max & Median & Mean & St.Dev \\
\hline PRE & SE & 1 & 8 & 4 & 4.16 & 1.57 \\
& PAR & 1.0 & 7 & 3 & 3.48 & 1.36 \\
& KS & 2 & 9 & 4 & 4.58 & 1.73 \\
& PC & 2 & 8 & 4 & 43 & 1.45 \\
& FR & 3 & 9 & 5 & 5.10 & 1.56 \\
& COM & 2 & 9 & 5 & 5.23 & 1.75 \\
POST & MOT & 1 & 8 & 4 & 4.065 & 1.65 \\
& SE & 3 & 9 & 5 & 5.58 & 1.76 \\
& PAR & 1 & 9 & 6 & 5.90 & 2.04 \\
& KS & 4 & 9 & 7 & 7 & 1.65 \\
& PC & 4 & 9 & 8 & 7.01 & 1.50 \\
& FR & 2 & 9 & 7 & 6.36 & 1.59 \\
& COM & 3 & 9 & 7 & 6.581 & 1.523 \\
& MOT & 4 & 9 & 7 & 6.64 & 1.60 \\
\hline
\end{tabular}


Table 15 Summary of the results of the Capacity-Belief Questionnaire

\begin{tabular}{lll}
\hline Variable & p-value & Effect size \\
\hline Self-Efficacy & 0.003 & -0.426 (medium) \\
Partnership & $5.687968 \mathrm{e}-06$ & -0.663 (large) \\
Knowledge \& Skills & $4.422434 \mathrm{e}-06$ & -0.671 (large) \\
Personal Control & $1.964025 \mathrm{e}-08$ & -0.819 (large) \\
Feeling Respected & 0.002 & -0.455 (medium) \\
Communication & 0.002 & -0.445 (medium) \\
Motivation & $7.604048 \mathrm{e}-07$ & -0.724 (large) \\
\hline
\end{tabular}

\subsection{Results}

In the following we report the results related to the three research questions related to Empowerment, Usability and User Experience.

\subsubsection{RQ1: empowerment}

As shown in Fig. 9, the empowerment beliefs perceived by the participants appear to increase after the experiment. Deeper information is reported in Table 14, where the summary statistics of the Empowerment variables distribution are shown. Table 15 reports the statistical significance and the Cliff Effect size of each variable. In all the cases there is a significant statistical difference with a medium or large effect size.

\subsubsection{RQ2: usability}

Effectiveness. All the participants successfully completed all the tasks.

Interactiveness. The distributions of the number of interactions appear similar in the case of SU and NSU, as shown in Fig. 10a. This is also confirmed by the statistical data in

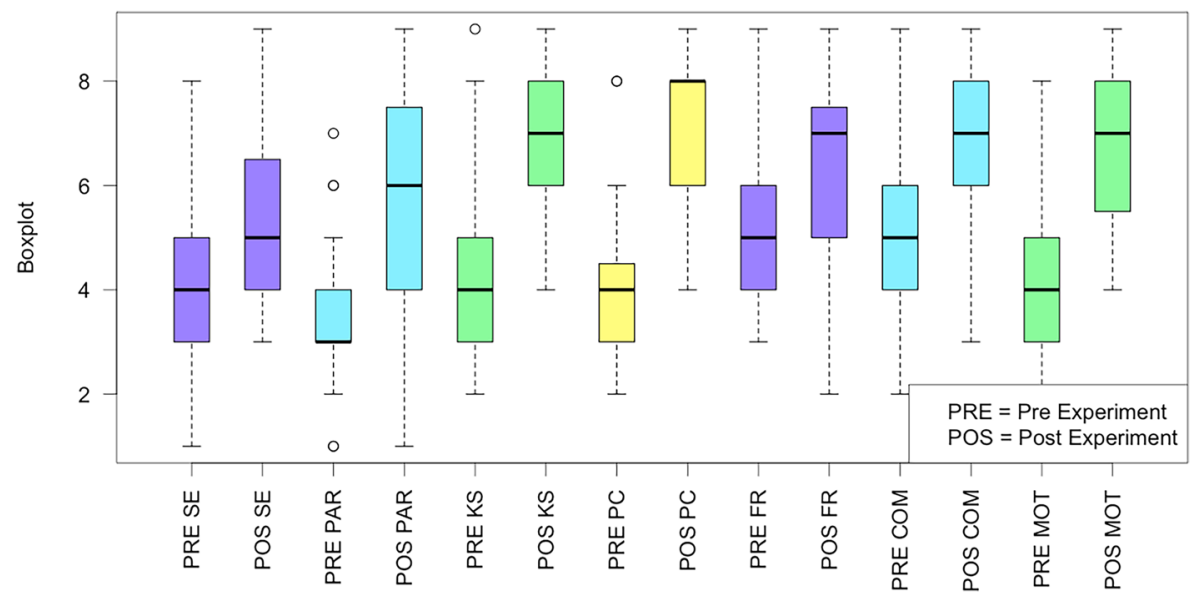

Fig. 9 Boxplots of the Capacity-Belief Questionnaire 
Table 16 Statistics of the Usability variables

\begin{tabular}{lllllll}
\hline Technology & Variable & Min & Max & Median & Mean & St.Dev \\
\hline NSU & click & 17 & 25 & 22 & 21.39 & 2.29 \\
& Time & 173 & 250 & 210 & 212.308 & 21.616 \\
\multirow{4}{*}{ SU } & Satisfaction & 3 & 7 & 5 & 5.15 & 1.28 \\
& click & 17 & 25 & 20.50 & 20.50 & 2.41 \\
& Time & 178 & 230 & 197 & 197.44 & 13.544 \\
\multirow{4}{*}{ Overall } & Satisfaction & 4 & 7 & 7 & 6.17 & 1.04 \\
& click & 17 & 25 & 21 & 20.87 & 2.36 \\
& Time & 173 & 250 & 199 & 199 & 13.67 \\
& Satisfaction & 3 & 7 & 6 & 5.74 & 1.24 \\
\hline
\end{tabular}

Table 16, where the summary statistics of each usability variable are reported for NSU and SU. The overall data of the sample is also resumed. Table 17 shows that the difference is not significative ( $\mathrm{p}$-value $=0.638)$.

Speed. The time to accomplish the task appear to be larger in the case of NSU users than SU ones, as shown in Fig. 10b. This is also confirmed by the statistics data in Table 17 and by the results of the statistical significance Mann-Whitney $U$ test ( $p$-value $=0.025$ ) with a large effect size.

Satisfaction. As shown in Table 16 and by the boxplot in Fig. 10c SU appears more satisfied than NSU. This is confirmed by the significant statistical difference highlighted in Table 17 (p-value=0.029) with medium effect size $(-0.444)$.

\subsubsection{RQ3: user experience}

Concerning the User Experience, the perception is generally positive, as shown in the boxplot in Fig. 11. Statistical analysis revealed only a significant difference in the perception of Efficiency ( $p$-value=0.036), with medium effect size (Cliff delta=-0.432).

\subsubsection{Threats to validity}

We report the treats to validity may affect the experiment results.

Internal validity. Low user experience. All the participants were new to the tool. It may be they did not understand the procedure. But generally, the Post Task questionnaire got a good result. In addition, we let participants practice with the tool before starting the experiment. The voluntary participation in the study may be a threat (motivation). All the participants were final end-users (caregivers) of a child with ASD and then motivated, as the target users should be. To limit the threat of diffusion or treatment imitations, the supervisor retained the material to avoid exchange of information by the users. Also a small sample size may be a threat to validity.

Table 17 Summary of the results of the Usability variables

\begin{tabular}{llc}
\hline Variable & p-value & Effect size \\
\hline Click & 0.638 & - \\
Time & 0.025 & 0.483 (large) \\
Satisfaction & 0.029 & -0.444 (medium) \\
\hline
\end{tabular}




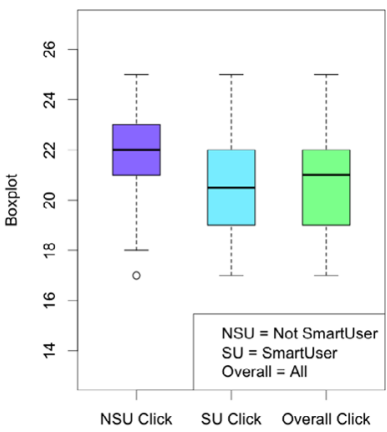

(a) Click

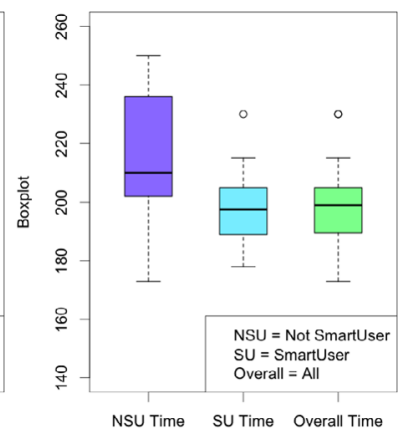

(b) Time

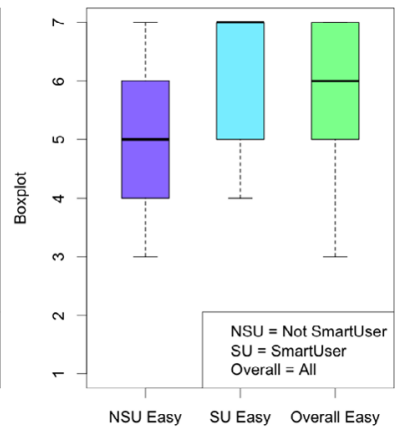

(c) Satisfaction

Fig. 10 Boxplot of Click (a), Time (b) and Satisfaction (c) variables

Construct validity. We also tried to mitigate evaluation apprehension threat by assuring participants that their information will be anonymously managed and diffused in aggregated form. A threat may be due to the absence of a baseline tool. To mitigate the restricted generalizability across constructs we considered the impact on the user smartphone use. The approach may impact other constructs we did not observe.

Conclusion validity. We choose tests that do not require normally distributed data, such as Mann-Whitney U test and Cliffs Delta effect size. The treatment was maintained similarly for the different participants: with the same setting and light.

External validity. To mitigate the external validity we considered both users with high and low familiarity with the use of smartphones. All the participants were target users of the application. Moreover, they were not informed on the aim of the study or on the research hypotheses.

\section{Discussion}

We discuss in the following the implications of our study and conclude this section by summarizing guidelines that may be useful for developing an empowering application for the TA of a different type of patients.

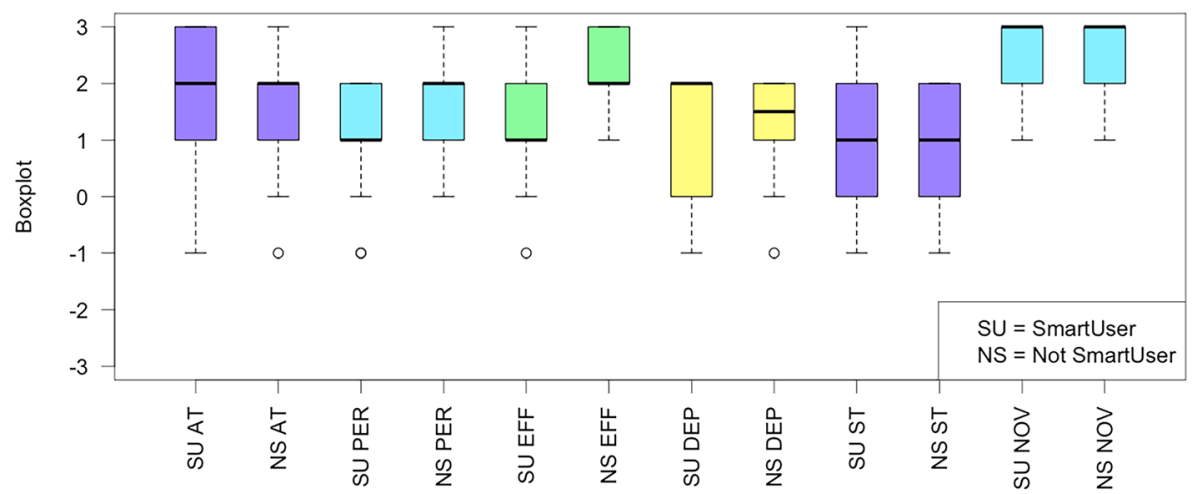

Fig. 11 Boxplots of the UEQ Questionnaire 


\subsection{Implications and future extensions}

We delineate a number of practical implications from the perspective of the different stakeholders and of the researchers to continue this work.

In this paper, we have described our work for empowering the therapeutic alliance members of a person with ASD. The aim is not a substitution of the physical relationships between the TA members, but their enhancement through a multimedia-based social sharing of methods, good practices, signs of progress, and recording of challenging behaviors. Previous researches concerned the sub-ministration of ABA therapy via tele-health, but our approach enables the increasing of empowerment beliefs of the patient's circle, by enhancing their communication, autonomy, awareness, and knowledge.

Overall, the results of the third iteration of the prototyping phase suggest that there was a positive attitude towards the application. In particular, the empowerment perceptions of the caregivers increased for all the variables after the tool has been used. This is mainly due to the absence of a similar application. Indeed, as an example, an open comment was: "When will this application be available?", or "Where can I download the application?". Open comments were quite enthusiastic, and confirmed our perception of the need of this kind of application.

The technological skills of the TA members may be a possible bias. Indeed, according to EU General Data Protection Regulation (GDPR) ${ }^{3}$ Thea should respect the right of Justice., e.g., the equal right of accessing to mHealth. To pursue this objective the application should be easy-to-use, robust, interoperable with the other healthcare systems, and accessible by all the citizens. Following this direction, in the user study in Sect. 6.4 we examined whether the application was easy to use for both smart skilled users (SU) and occasional smart users (NSU). We also add the interaction with a chat-bot for guiding the user in the selection of the app functionalities. Preliminary results were encouraging: also occasional smart users completed all the tasks, they do not have particular differences with the interaction with the application, but took more time. This is also revealed by the UX data related to efficiency: the system is perceived as more efficient by SU, even if the average values are both positive (1.782 for SU vs 1.0 for NSU). Similar consideration holds for the easyto-use question after each task (6.17 was the average for SU, and 5.15 for NSU). Thus, the user ability in using smartphones impacts task performances. The supervisor revealed that a user had a problem using the chatbot because of her pronunciation. A training of Thea on the user pronunciations has to be inserted. UX results were positive, especially for the novelty perception, but the interaction with Thea should be further simplified for increasing efficiency.

Thea may help to solicit therapists in recording their sessions by videos, a much-felt need by the relatives and the teachers. Indeed, the therapist may take videos in a easier way, in hand-free modality. This may be enforced by recommending to add rewards both for health providers and patients/caregivers in terms of points and showing them on a community board. The adding of reputation features may motivate the health providers in sharing their competencies through video content.

It is also worth mentioning that teachers and caregivers may be stimulated by the social aspects to produce examples of their work and the signs of progress of the child, with a virtuous mechanism of emulation. Concerning the clinician, she may better supervise the

\footnotetext{
3 https://europa.eu/youreurope/business/dealing-with-customers/data-protection/data-protection-gdpr/ index_en.htm
} 
work of therapists, the family involvement, and the child progress, having all this information in a unique place in a classified and organized way. All the stakeholders benefit from the possibility of recording activities and challenging behaviors in a hand-free modality. It is very difficult to take a video in a challenging situation. On the other side, all the TA members are solicited to contribute to filling in the electronic Diary of all the activities, organized by tasks.

Because the therapy for people with ASD requires to be applied for several hours a day, also patients and their circle living in rural and remote areas far from specialists or in the pandemic situation in which the distance is counseled may benefit from this kind of distance counseling. In particular, during the Covid-19 pandemic context children with ASD have to stay at home. They should continue to receive an education. Thea provides valid support to all the stakeholders for this situation.

The use of Thea has the secondary (but very relevant) effect to reduce challenging behaviors by teaching caregivers and teachers to prevent and manage them. This improves the quality of life of all the family, with a positive perception of empowerment and the reduction of the feeling of abandonment of everybody, including teachers. The multimedia contents collected by THEA also provide a trace of the progress and contribute to motivating the circle.

In Italy people with autism fall under the care of the State. School is free for all the children. One-to-one educational support is provided to children with ASD at school, depending on the ASD severity level. ABA interventions are not offered by the Italian health system which provides few hours per weeks of psychomotor therapy, or logotherapy [9]. In Italy, the practice of online counseling in the public field is absent [7]. Nevertheless, there is an increasing demand of ABA services, which are mainly privately funded by the families and performed at home after school. The communication approach supported by THEA creates a network among the circle people, involving also the teacher and the patient when enabled by her autonomy level. Thus the therapy may be applied everywhere (school or home), with a sense of community. Our system enables the structuring of the activity to perform with the possibility of organizing activities for objectives, such as learning to be autonomous in primary needs (e.g., breakfast, washing, dressing). Thus, caregivers and teachers may easier involve the child in structured activities different from her preferred solitary activities.

Another advantage of Thea is that when a teacher, a clinician or a therapist changes, her substitute may in a short time be informed on the child therapy and his challenging problems.

Concerning the end-user interaction, our study adopted a chatbot as interface. Both researchers and practitioners may be interested in investigating whether the use of other interaction modalities, such as the gestures, may improve the empowering perceptions of the target users. Also the use of wearable technologies [13] may be integrated in Thea for detecting or predicting challenging behaviours.

Patient empowerment requires the patient to be at the center of the process. In the case of children with autism, this is not always possible because the autism spectrum has several gravity levels. The circles we examined in this case study were related to patients who did not have such an autonomy level and were not able to perform activities without support. For this reason, the application developed and described in this paper seems more oriented to child relatives and caregivers. We have to extend it by examining the usability requirements also for more autonomous children. 
Because the current experiment has been conducted with parents of children with ASD further studies involving the use of the system by a large sample of all the stakeholders are needed to further improve the easiness-to-use of the system.

The diffusion of a new technology/method is easier when user studies empirically show that it solves real issues [33]. The results of our study suggest that the functionalities offered by Thea may be very useful for the community of people with ASD. This outcome could increase the diffusion of such a kind of empowering approaches and the definition of new ones for other target of patients. These points are clearly relevant for both the practitioner and the researcher.

\subsection{Guidelines}

This study may provide useful guidelines to identify inefficiencies and factors to be improved in the case there is the need of developing an application for enhancing the TA for a different kind of pathology to be inserted in a user-centered design process. In the following we summarize some guidelines that can be drawn by this study.

- Guideline 1: identify the needs for actuating the behavior changes. This may be performed by understanding the empowerment levels of the Therapeutic Alliance members and of the provider(s) of the health service. Based on the empowerment goals summarized in Sect. 5 the tools developed in this paper may be applied: the template document shown in Table 2 and Table 3 may be adopted to customize the TAT for conducting a discussion with representative users, i.e., caregivers and clinicians, respectively.

- Guideline 2: identify the empowerment objectives and the multimedia interaction needs by using task analysis. Analyze for each individuated task the behaviors and the capability to empower and whether the multimedia technology may support the task execution, as shown in Table 6.

- Guideline 3: design the empowerment process by using a scenario-based description supported by a model of the process represented by an activity diagram with objectflows and swimlanes. The $<<$ Multimedia $>>$ stereotype may be adopted for signaling the activities requiring a more specific multimedia interaction design.

- Guideline 4: assess the user perception of empowerment. The assessment of the Empowerment perception before and after using the application may be useful to understand whether the application increases the capacities and the beliefs of the TA members in the management of the patient's condition. The questionnaire adopted in this paper (shown in Table 12 and validated in Appendix A) may be adopted.

\section{Conclusion}

In this paper, we presented an approach for supporting the empowerment of the Therapeutic Alliance of children with ASD through the definition of communication process among the alliance members with the adoption of multimedia interaction technologies. We identified the aspects that should be empowered in terms of capacities and behaviors by exploiting TAT. When evaluating the application we considered two kinds of smartphone users: 
expert and occasional. The results collected in the case study are preliminary, but encouraging, also for occasional users.

Multimedia applications for the empowerment of TA may be a solution for rehabilitation problems where there is a shortage of therapists and clinicians. This is particularly true in our specific contingent moment of the Coronavirus pandemic. Indeed, children with ASD stay at home, and their family members need to keep on providing the needed care to them in order not to lose the acquired abilities.

Future work may be devoted to collect all the content produced by the multimedia applications and the success stories to create a knowledge base useful for the patient community. Recommendation systems could be designed to suggest training activities to patients, under suitable safety assurance and medical control.

\section{Appendix A}

Before using the data collected by the questionnaire reported in Table 12 to assess the Empowerment perception in our study, we studied its reliability. We derived it from established questionnaire [22]. Further verification was needed to understand in which measure the applied modifications preserve the reliability of the initial questionnaire. To this aim, we applied the Principal Components Analysis (PCA) on the data collected before starting the experiment for examining the factor structure of the questionnaire. Then we verified the internal reliability of the questionnaire by using Cronbach's $\alpha$. The usual threshold for acceptability for Cronbach's $\alpha$ is 0.7 [31].

As shown in Fig. 12, the components PC1-PC5 reached a cumulative proportion of $95 \%$ of variance. Thus, PC6 and PC7 could be excluded. We may select the variable reaching variance greater equal than 0.5, i.e., SE and MOT for PC1, FR and COM for PC2, KS and PAR for PC3, and PC for PC4. Cronbach's $\alpha$ was 0.99 at $95 \%$ of confidence level for the selected questions in PC1; for FR and COM it was 0.58. So we assigned COM to PC6 and FR to PC2. A similar result is reached for PC3 (Cronbach's $\alpha=0.25$ ). We assigned KS to PC5. As we observed that SE also reach 0.70 in PC7, we decide that the questions we extracted from [22] may be used without grouping them in factors (see Fig. 13).

$\begin{array}{lrrrrrrr} & \text { PC1 } & \text { PC2 } & \text { PC3 } & \text { PC4 } & \text { PC5 } & \text { PC6 } & \text { PC7 } \\ \text { Standard deviation } & 1.4213 & 1.2845 & 1.0597 & 0.9833 & 0.9488 & 0.57150 & 0.11550 \\ \text { Proportion of Variance } & 0.2886 & 0.2357 & 0.1604 & 0.1381 & 0.1286 & 0.04666 & 0.00191 \\ \text { Cumulative Proportion } & 0.2886 & 0.5243 & 0.6847 & 0.8228 & 0.9514 & 0.99809 & 1.00000\end{array}$

Fig. 12 Importance of Components

\begin{tabular}{lrrrrrrr|r|} 
& PC1 & PC2 & PC3 & PC4 & PC5 & PC6 & PC7 \\
SE & 0.696813010 & -0.016303608 & 0.08521075 & -0.01293484 & 0.06381955 & -0.01400686 & 0.70886424 \\
PAR & -0.019629268 & -0.133807249 & 0.71627152 & 0.28958516 & -0.57081868 & -0.24217376 & -0.01799302 \\
KS & -0.183064165 & -0.153263005 & 0.57663900 & 0.13042853 & 0.73583763 & 0.22303880 & 0.04764979 \\
PC & 0.034764013 & -0.166706956 & -0.33611813 & 0.92122715 & 0.04799096 & 0.08239870 & 0.01651409 \\
FR & -0.006276746 & 0.677692288 & 0.04565443 & 0.18442316 & 0.27120427 & -0.65630076 & -0.01775102 \\
COM & -0.012423875 & 0.686499133 & 0.14384745 & 0.12692635 & -0.20136383 & 0.67025630 & 0.04439931 \\
MOT & 0.692207733 & -0.001076052 & 0.10691065 & 0.01341106 & 0.11060648 & 0.06815890 & -0.70168235 \\
\hline
\end{tabular}

Fig. 13 Rotation $(n \times k)=(7 \times 7)$ 
Funding Open access funding provided by Università degli Studi di Salerno within the CRUI-CARE Agreement.

Open Access This article is licensed under a Creative Commons Attribution 4.0 International License, which permits use, sharing, adaptation, distribution and reproduction in any medium or format, as long as you give appropriate credit to the original author(s) and the source, provide a link to the Creative Commons licence, and indicate if changes were made. The images or other third party material in this article are included in the article's Creative Commons licence, unless indicated otherwise in a credit line to the material. If material is not included in the article's Creative Commons licence and your intended use is not permitted by statutory regulation or exceeds the permitted use, you will need to obtain permission directly from the copyright holder. To view a copy of this licence, visit http://creativecommons.org/licenses/by/4.0/.

\section{References}

1. Abrahamyan S, Balyan S, Muradov A, Kulabukhova N, Korkhov V (2017) A concept of unified e-health platform for patient communication and monitoring. In Computational Science and Its Applications, pages 448-462. Springer International Publishing

2. Aguilera A, Muench F (2012) There's an app for that: Information technology applications for cognitive behavioral practitioners. The Behavior therapist/AABT 35(4):65

3. Anderson RM, Funnell MM, Butler PM, Arnold MS, Fitzgerald JT, Feste CC (1995) Patient empowerment: results of a randomized controlled trial. Diabetes care 18(7):943-949

4. Bandura A (1986) Social foundations of action: A social - cognitive theory. Englewood Cliffs, 617

5. Bravo P, Edwards A, Barr PJ, Scholl I, Elwyn G, McAllister M (2015) Conceptualising patient empowerment: a mixed methods study. BMC Health Serv Res 15(1):252

6. Castonguay LG, Goldfried MR, Wiser S, Raue PJ, Hayes AM (1996) Predicting the effect of cognitive therapy for depression: a study of unique and common factors. J Consult Clin Psychol 64(3):497

7. Cipolletta S, Mocellin D (2018) Online counseling: An exploratory survey of italian psychologists' attitudes towards new ways of interaction. Psychother Res 28(6):909-924

8. De Bell S, Graham H, Jarvis S, White P (2017) The importance of nature in mediating social and psychological benefits associated with visits to freshwater blue space. Landsc Urban Plan 167:118-127

9. Degli Espinosa F, Metko A, Raimondi M, Impenna M, Scognamiglio E (2020) A model of support for families of children with autism living in the covid-19 lockdown: Lessons from italy. Behav Anal Pract 13:550-558

10. Eldevik S, Hastings RP, Hughes JC, Jahr E, Eikeseth S, Cross S (2009) Meta-analysis of early intensive behavioral intervention for children with autism. J Clin Child Adolesc Psychol 38(3):439-450

11. Feinstein NR, Fielding K, Udvari-Solner A, Joshi SV (2009) The supporting alliance in child and adolescent treatment: Enhancing collaboration among therapists, parents, and teachers. Am J Psychiatry 63(4):319-344

12. Ferreira JM, Acuña ST, Dieste O, Vegas S, Santos A, Rodríguez F, Juristo N (2020) Impact of usability mechanisms: An experiment on efficiency, effectiveness and user satisfaction. Inf Softw Technol 117

13. Francese R, Yang X (2021) Supporting autism spectrum disorder screening and intervention with machine learning and wearables: a systematic literature review. Complex \& Intelligent Systems 1-16

14. Freeman RL, Britten J, McCart A, Smith C, Poston D, Anderson D, Edmonson H, Baker D, Sailor W, Guess D, Reichle J (2020) Functional Assesment. http://www.specialconnections.ku.edu/ specconn/page/ behavior/fba/pdf/completedabcchart.pdf. [Online; accessed 10-April-2020]

15. Freire $\mathrm{P}$ (2018) Pedagogy of the oppressed. Bloomsbury publishing

16. Freund PD (1993) Professional role(s) in the empowerment process: working with mental health consumers. Psychiatr Rehabil J 16(3):65

17. Green J (2006) Annotation: The therapeutic alliance-a significant but neglected variable in child mental health treatment studies. J Child Psychol Psychiatry 47(5):425-435

18. Hess DJ (1996) The ethics of compliance: A dialectic. Adv Nurs Sci 19(1):18-27

19. Hill JH (2016) The impact of emojis and emoticons on online consumer reviews, perceived company response quality, brand relationship, and purchase intent. In Thesis

20. Horvath AO, Luborsky L (1993) The role of the therapeutic alliance in psychotherapy. J Consult Clin Psychol 61(4):561

21. Hougaard E (1994) The therapeutic alliance-a conceptual analysis. Scand J Psychol 35(1):67-85

22. Kim SC, Boren D, Solem SL (2001) The kim alliance scale: development and preliminary testing. Clin Nurs Res 10(3):314-331 
23. Lamprinos I, Demski H, Mantwill S, Kabak Y, Hildebrand C, Ploessnig M (2016) Modular ict-based patient empowerment framework for self-management of diabetes: Design perspectives and validation results. J Med Inform 91:31-43

24. Law GC, Dutt A, Neihart M (2019) Increasing intervention fidelity among special education teachers for autism intervention: A pilot study of utilizing a mobile-app-enabled training program. Res Autism Spectr Disord 67

25. Lindgreen P, Clausen L, Lomborg K (2018) Clinicians' perspective on an app for patient self-monitoring in eating disorder treatment. J Eat Disord 51(4):314-321

26. Macbeth G, Razumiejczyk E, Ledesma RD (2011) Cliff's delta calculator: A non-parametric effect size program for two groups of observations. Universitas Psychologica 10(2):545-555

27. McAllister M, Dunn G, Payne K, Davies L, Todd C (2012) Patient empowerment: the need to consider it as a measurable patient-reported outcome for chronic conditions. BMC Health Serv Res 12(1):157

28. McDougall T (1997) Patient empowerment: Fact or fiction? Mental Health Nursing-London-Community Psychiatric Nurses Association 17:4-7

29. Miles MB, Huberman AM, Huberman MA, Huberman M (1994) Qualitative data analysis: An expanded sourcebook. Sage

30. Muran JC, Barber JP (2011) The therapeutic alliance: An evidence-based guide to practice. Guilford Press

31. Nunnally JC (1975) Psychometric theory-25 years ago and now. Educ Res 4(10):7-21

32. Pekonen A, Eloranta S, Stolt M, Virolainen P, Leino-Kilpi H (2019) Measuring patient empowerment-a systematic review. Patient Educ Couns

33. Pfleeger SL, Menezes W (2000) Marketing technology to software practitioners. IEEE Software 17(1):27-33

34. Rahman MS, Ko M, Warren J, Carpenter D (2016) Healthcare technology self-efficacy (htse) and its influence on individual attitude: An empirical study. Comput Hum Behav 58:12-24

35. Rauschenberger M., Schrepp M, Pérez Cota M, Olschner S, Thomaschewski J (2013) Efficient measurement of the user experience of interactive products. how to use the user experience questionnaire (ueq). example: Spanish language version. J Interac Multimed Artif Intell

36. Richards P, Simpson S, Bastiampillai T, Pietrabissa G, Castelnuovo G (2018) The impact of technology on therapeutic alliance and engagement in psychotherapy: The therapist's perspective. Clin Psychol 22(2):171-181

37. Schnall R, Rojas M, Bakken S, Brown W, Carballo-Dieguez A, Carry M, Gelaude D, Mosley JP, Travers J (2016) A user-centered model for designing consumer mobile health (mhealth) applications (apps). J Biomed Inform 60:243-251

38. Sun V, Raz DJ, Ruel N, Chang W, Erhunmwunsee L, Reckamp K, Tiep B, Ferrell B, McCorkle R, Kim JY (2017) A multimedia self-management intervention to prepare cancer patients and family caregivers for lung surgery and postoperative recovery. Clin Lung Cancer 18(3):e151-e159

39. Tarasewich P, Gong J, Nah F (2007) Interface design for handheld mobile devices. AMCIS 2007 Proceedings, pages 352

40. Vitiello G, Francese R, Sebillo M, Tortora G, Tucci M (2017) Ux-requirements for patient's empowerment - the case of multiple pharmacological treatments: A case study of IT support to chronic disease management. In Requirements Engineering, pages 139-145

41. Vitiello G, Francese R, Sebillo M, Tortora G, Tucci M (2017) Ux-requirements for patient's empowerment the case of multiple pharmacological treatments: A case study of it support to chronic disease management. In Requirements Engineering, pages 139-145

42. Vitiello G, Sebillo M (2018) The importance of empowerment goals in elderly-centered interaction design. In Advanced Visual Interfaces, pages 1-5

Publisher's Note Springer Nature remains neutral with regard to jurisdictional claims in published maps and institutional affiliations. 


\section{Authors and Affiliations}

Rita Francese $^{1}$ D $\cdot$ Michele Risi $^{1} \cdot$ Genoveffa Tortora $^{1} \cdot$ Francesco Di Salle $^{1}$

Michele Risi

mrisi@unisa.it

Genoveffa Tortora

tortora@unisa.it

Francesco Di Salle

fdisalle@unisa.it

1 Department of Computer Science, University of Salerno, Fisciano, Italy 